\title{
Numerical Evaluation of the Accuracy and Stability Properties of High-order Direct Stokes Solvers with or without Temporal Splitting
}

\author{
E. Leriche, ${ }^{1}$ E. Perchat, ${ }^{2}$ G. Labrosse,${ }^{3}$ and M.O. Deville ${ }^{4}$
}

Received January 15, 2004; accepted (in revised form) August 20, 2004

\begin{abstract}
The temporal stability and effective order of two different direct high-order Stokes solvers are examined. Both solvers start from the primitive variables formulation of the Stokes problem, but are distinct by the numerical uncoupling they apply on the Stokes operator. One of these solvers introduces an intermediate divergence free velocity for performing a temporal splitting (J. Comput. Phys. [1991] 97, 414-443) while the other treats the whole Stokes problem through the evaluation of a divergence free acceleration field (C.R. Acad. Sci. Paris [1994] 319 Serie I, 1455-1461; SIAM J. Scient. Comput. [2000] 22(4), 1386-1410). The second uncoupling is known to be consistent with the harmonicity of the pressure field (SIAM J. Scient. Comput. [2000] 22(4), 1386-1410). Both solvers proceed by two steps, a pressure evaluation based on an extrapolated in time (of theoretical order $J_{e}$ ) Neumann condition, and a time implicit (of theoretical order $J_{i}$ ) diffusion step for the final velocity. These solvers are implemented with a Chebyshev mono-domain and a Legendre spectral element collocation schemes. The numerical stability of these four options is investigated for the sixteen combinations of $\left(J_{e}, J_{i}\right), 1 \leqslant J_{e}, J_{i} \leqslant 4$.
\end{abstract}

\footnotetext{
${ }^{1}$ Corresponding author, Laboratoire d'Ingénierie Numérique, Institut des Sciences de l'Energie, Section de Génie Mécanique, Faculté des Sciences et Techniques de l'Ingénieur, Ecole Polytechnique Fédérale de Lausanne, Station 9, CH-1015 Ecublens, Switzerland. E-mail: emmanuel.leriche@epfl.ch

${ }^{2}$ Laboratoire d'Ingénierie Numérique, Institut des Sciences de l'Energie, Section de Génie Mécanique, Faculté des Sciences et Techniques de l'Ingénieur, Ecole Polytechnique Fédérale de Lausanne, CH-1015 Ecublens, Switzerland, Present address: Transvalor SA, Parc de Haute Technologie, Sophia Antipolis, 694 av. du Dr Donat, 06255 Mougins cedex, France E-mail: eperchat@wanadoo.fr

${ }^{3}$ Université Paris-Sud, LIMSI-CNRS, BP 133, 91403 Orsay Cedex, France. E-mail: labrosse@limsi.fr

${ }^{4}$ Laboratoire d'Ingénierie Numérique, Institut des Sciences de l'Energie, Section de Génie Mécanique, Faculté des Sciences et Techniques de l'Ingénieur, Ecole Polytechnique Fédérale de Lausanne, CH-1015 Ecublens, Switzerland. E-mail: michel.deville@epfl.ch
} 
KEY WORDS: Stokes equations; decoupling methods; temporal splitting methods; spectral Chebyshev and Legendre approximation.

\section{INTRODUCTION}

The numerical simulation of incompressible fluid flows is performed by solving the Navier-Stokes equations. In the framework of the spectral approximations, the state-of-the-art time algorithms handle the pressure and the viscous terms implicitly through an unsteady Stokes problem, with the non-linear terms treated explicitly in time as source. This holds even for the direct numerical simulation of turbulent flows [15]. Solving accurately and efficiently the two-dimensional (2D) or three-dimensional (3D) Stokes system in primitive variables is therefore the corner stone of accurate numerical experiments. Among the numerous solvers available so far, the Uzawa and influence matrix (or Green functions) methods can be left aside for their well-known prohibitive costs.

In contrast, the fractional step methods $[4,18]$, based on a temporal splitting of the operators, are significantly cheaper. Their extensions to high-order accuracy in time has been established by Karniadakis et al. [12], leading to the hereafter referred to as high-order splitting (HOS) schemes. The pressure is evaluated through an extrapolated in time (of theoretical order $J_{e}$ ) Neumann boundary condition, whereas the velocity is obtained by a time implicit (of theoretical order $J_{i}$ ) diffusion step. However the HOS schemes are known to be inconsistent with the continuous Stokes problem $[12,16]$. Recently, Guermond and Shen $[9,11,10]$ reformulated the HOS and provided a first rigorous proof of stability and convergence of the HOS limited to $J_{i}=1,2$ and $J_{e}=1$ time schemes.

A non-splitting and therefore consistent formulation (hereafter referred to as HONS) of the HOS scheme is proposed in this paper. This method gathers the advantages of the HOS scheme (high-order accuracy) with the consistency of the projection-diffusion scheme proposed in [2]. In both schemes the pressure step calls for an explicit in time boundary condition (of order $J_{e}$ ) and is followed by an implicit in time (of order $J_{i}$ ) diffusion step for the velocity. A numerical investigation of the comparative properties of the HOS schemes and projection-diffusion schemes [2] has been initiated in [16], but only in the case where the temporal integration orders are the same, $J_{e}=J_{i}$, up to the fourth order. A numerical evaluation of the stability and time accuracy properties, with any combination $1 \leqslant\left(J_{e}, J_{i}\right) \leqslant 4$, is performed here for both HOS and HONS schemes with two spectral space integration approaches : a Chebyshev collocation method (hereafter denoted by CCM), and a Legendre spectral element method (SEM). 
The paper is organized as follows : the governing equations are given in Sec. 2, then the HOS and HONS Stokes solvers in their continuous-in-space formulation are briefly presented in Sec. 3. Section 4 contains the main features of the space discretisation. The stability and the effective time accuracy of the HOS and HONS schemes are successively reported in Secs. 5 and 6.

\section{THE GOVERNING EQUATIONS}

Let us consider the dimensionless unsteady (time $t$ ) two- or threedimensional Stokes equations, written in the open domain $\Omega$ with coordinates $\mathbf{x}=\left(x_{i}, i=1, d\right), \equiv(x, y)$ or $(x, y, z)$ with $d=2,3$, respectively, $d$ being the space dimension and $T$ a real positive number:

$$
\begin{aligned}
\frac{\partial \mathbf{u}}{\partial t} & =\Delta \mathbf{u}-\nabla p+\mathbf{f} \quad \text { for }(\mathbf{x}, t) \in \Omega \times] 0, T[, \\
\nabla \cdot \mathbf{u} & =0 \quad \text { for }(\mathbf{x}, t) \in \Omega \times] 0, T[,
\end{aligned}
$$

where $\mathbf{u}=(u, v, w)$ is the velocity field, $p$ the pressure and $\mathbf{f}$ the source term including the non-linear advective terms beside possible prescribed body forces. We denote the closure of $\Omega$ by $\bar{\Omega}$ and the boundary by $\partial \Omega$. For the sake of simplicity, we consider Dirichlet boundary conditions

$$
\mathbf{u}=\mathbf{U} \text { for }(\mathbf{x}, t) \in \partial \Omega \times] 0, T[
$$

and compatible initial conditions are given,

$$
\mathbf{u}(t=0)=\mathbf{U}^{0} \quad \text { for } \mathbf{x} \in \Omega,
$$

$\mathbf{U}^{0}$ being divergence-free.

\section{THE STOKES SOLVERS}

The unsteady Stokes problem is now solved with two different $(\mathbf{u}, p)$ decoupling schemes. The first one, denoted by HOS, is the well established fractional step algorithm [12]. It is briefly summarized in the beginning of this section. Basically linked to a time scheme, its presentation is made in a semi-discrete framework, i.e., discretised in time and continuous in space. The second one, the HONS, is then presented in more detail.

\subsection{The HOS Method}

Proposed in 1991 as a high-order splitting method for the NavierStokes equations, the HOS time discretised system, applied to the unsteady Stokes problem, uses the backward Euler scheme of order $J_{i}$. Its continuous-in-space version reads: 


$$
\begin{aligned}
\frac{\hat{\mathbf{u}}-\sum_{q=0}^{J_{i}-1} \alpha_{q} \mathbf{u}^{n-q}}{\Delta t} & =-\nabla p^{n+1}+\mathbf{f}^{n+1} & & \text { for } \mathbf{x} \in \Omega, \\
\nabla \cdot \hat{\mathbf{u}} & =0 & & \text { for } \mathbf{x} \in \Omega, \\
\frac{\gamma_{0} \mathbf{u}^{n+1}-\hat{\mathbf{u}}}{\Delta t} & =\Delta \mathbf{u}^{n+1} & & \text { for } \mathbf{x} \in \Omega, \\
\mathbf{u}^{n+1} & =\mathbf{U}^{n+1} & & \text { for } \mathbf{x} \in \partial \Omega,
\end{aligned}
$$

where $\hat{\mathbf{u}}$ is an intermediate velocity field, constrained to fulfil the incompressibility condition (3.2), and $\mathbf{u}^{n} \equiv \mathbf{u}(n \Delta t)$. Equations (3.1) and (3.2) are combined to lead to the Poisson problem for the pressure, with boundary conditions obtained from a time extrapolation of order $J_{e}$ proposed in [12, 17] and analysed in [16]:

$$
\begin{gathered}
\boldsymbol{\Delta} p^{n+1}=\nabla \cdot\left(\frac{\sum_{q=0}^{J_{i}-1} \alpha_{q} \mathbf{u}^{n-q}}{\Delta t}+\mathbf{f}^{n+1}\right) \quad \text { for } \mathbf{x} \in \Omega, \\
\frac{\partial p^{n+1}}{\partial \mathbf{n}}=\left(-\left(\frac{\partial \mathbf{U}}{\partial t}\right)^{n+1}-\sum_{q=0}^{J_{e}-1} \beta_{q} \nabla \times\left(\nabla \times \mathbf{u}^{n-q}\right)+\mathbf{f}^{n+1}\right) \cdot \mathbf{n} \text { for } \mathbf{x} \in \partial \Omega,
\end{gathered}
$$

with $\mathbf{n}$ being the outward normal unit vector. As explained in [16], the $-\nabla \times \nabla \times \mathbf{u}$ solenoidal term in the pressure boundary condition is chosen instead of $\boldsymbol{\Delta} \mathbf{u}$ in order to preserve the ellipticity of the Stokes problem. The irrotational part $\nabla(\nabla \cdot \mathbf{u})$ is dropped in accordance with Eq. (2.2). The case $J_{e}=J_{i}$ has been analysed, up to the fourth order, in [16], where the weights $\gamma_{0}, \alpha_{q}$ and $\beta_{q}$ may be found. Any combination $1 \leqslant\left(J_{e}, J_{i}\right) \leqslant 4$ is considered in the present work.

\subsection{The HONS Method}

Here the decoupling is defined independently of any temporal scheme. An intermediate divergence free field is introduced, the acceleration $\mathbf{a}$,

$$
\mathbf{a}=\frac{\partial \mathbf{u}}{\partial t}-\boldsymbol{\Delta} \mathbf{u}
$$

The original problem (2.1) and (2.2) splits, for each $t \in] 0, T$, into two steps: 
(1) first step:

$$
\begin{array}{rlrl}
\mathbf{a}+\nabla p & =\mathbf{f} & & \text { for } \mathbf{x} \in \Omega, \\
\nabla \cdot \mathbf{a} & =0 & & \text { for } \mathbf{x} \in \Omega, \\
\frac{\partial p}{\partial \mathbf{n}} & =(-\mathbf{a}+\mathbf{f}) \cdot \mathbf{n} & \text { for } \mathbf{x} \in \partial \Omega .
\end{array}
$$

This projection step can be rewritten either as a quasi-Poisson problem (cf. [16]) or as a Poisson-Neumann problem similar to (3.5) and (3.6):

$$
\begin{array}{ll}
\boldsymbol{\Delta} p=\nabla \cdot \mathbf{f} & \text { for } \mathbf{x} \in \Omega, \\
\frac{\partial p}{\partial \mathbf{n}}=\left(-\frac{\partial \mathbf{U}}{\partial t}-\nabla \times(\nabla \times \mathbf{u})+\mathbf{f}\right) \cdot \mathbf{n} & \text { for } \mathbf{x} \in \partial \Omega .
\end{array}
$$

In this paper, the Poisson-Neumann problem is preferred because the quasi-Poisson problem is difficult to extend to the multi-domain spectral element case.

(2) Second step:

$$
\begin{aligned}
\frac{\partial \mathbf{u}}{\partial t}-\boldsymbol{\Delta} \mathbf{u} & =\mathbf{a} \text { for } \mathbf{x} \in \Omega, \\
\mathbf{u} & =\mathbf{U} \text { for } \mathbf{x} \in \partial \Omega .
\end{aligned}
$$

The two steps are now discretised in time and read as follows:

(1) first step:

$$
\begin{array}{lr}
\boldsymbol{\Delta} p^{n+1}=\nabla \cdot \mathbf{f}^{n+1} & \text { for } \mathbf{x} \in \Omega, \\
\frac{\partial p^{n+1}}{\partial \mathbf{n}}=\left(-\left(\frac{\partial \mathbf{U}}{\partial t}\right)^{n+1}-\sum_{q=0}^{J_{e}-1} \beta_{q} \nabla \times\left(\nabla \times \mathbf{u}^{n-q}\right)+\mathbf{f}^{n+1}\right) \cdot \mathbf{n} \text { for } \mathbf{x} \in \partial \Omega .
\end{array}
$$

(2) second step:

$$
\begin{array}{rlrl}
\frac{\gamma_{0} \mathbf{u}^{n+1}-\sum_{q=0}^{J_{i}-1} \alpha_{q} \mathbf{u}^{n-q}}{\Delta t}-\Delta \mathbf{u}^{n+1} & =\mathbf{a}^{n+1}=\mathbf{f}^{n+1}-\nabla p^{n+1} & & \text { for } \mathbf{x} \in \Omega, \\
\mathbf{u}^{n+1} & =\mathbf{U}^{n+1} & \text { for } \mathbf{x} \in \partial \Omega .
\end{array}
$$




\subsection{Comments}

Both schemes have been presented, the second one from its continuous- to the discrete-in-time formulations whereas the first one requires a time discretisation scheme form the start. In both schemes, the two stages are coupled only through the same normal boundary condition, (3.6) or (3.15). In the first steps (3.5), (3.6) and (3.14), (3.15) the pressure is computed through the solution of the Poisson-Neumann problem. It is to be noted that the part $\sum_{q=0}^{J_{i}-1} \alpha_{q} \mathbf{u}^{n-q} / \Delta t$ of the velocity time derivative $\partial \mathbf{u} / \partial t$ is a source term in the pressure Poisson equation for the HOS scheme. The second steps (3.3), (3.4) and (3.16), (3.17) supply the velocity field by solving a vectorial elliptic problem. They are formally identical but, fed by the pressure gradient, they lead to different velocity fields for the reason mentioned just above. This is the essential cause of the inconsistency of the HOS scheme with respect to the following continuous uncoupled problem (harmonic pressure and biharmonic velocity fields, [12]),

$$
\left.\boldsymbol{\Delta} p=\nabla \cdot \mathbf{f}, \quad\left(\frac{\partial}{\partial t}-\boldsymbol{\Delta}\right) \boldsymbol{\Delta} \mathbf{u}=0, \quad \text { for }(\mathbf{x}, t) \in \Omega \times\right] 0, T[
$$

as fully investigated in [16].

\section{SPACE DISCRETISATION}

Two spectral space approximations have been adopted, a mono-domain CCM, and a multi-domain Legendre SEM. The former deals only with simple orthogonal geometry (Cartesian in the present paper), whereas the latter takes advantage of the finite element flexibility to get accurate numerical flow simulation in complex geometries, partitioned into elements [6]. Both spectral discretizations proceed by expanding, either in the domain or in each element, the $\mathbf{u}$ and $p$ fields in tensor product of high-order Lagrangian polynomials, $(N, M, L)$ for the $(x, y, z)$ dependencies, respectively. In the present study, $N, M$, and $L$ are equal, and denoted by $N$. The CCM consists of exactly enforcing the differential equations, and the boundary conditions, at the Gauss-Lobatto-Chebyshev points [3,8]. The SEM is based on a Galerkin formulation of the differential equations, using Gauss-LobattoLegendre interpolants [6]. For the SEM computations the object-oriented spectral element toolbox SPECULOOS is used [7].

For the sake of comparison of the CCM and SEM numerical properties, the same geometry has been adopted, the Cartesian domain $([-1,+1])^{d}$, and the same number of nodes in each space direction. Moreover the same number of elements, $E$, has been taken in each space direction. 
In both space discretisations applied to HOS and HONS schemes, the velocity $\mathbf{u}$ and pressure $p$ fields are approximated in the same polynomial space. These solvers are therefore of $\left(\mathbb{P}_{N}, \mathbb{P}_{N}\right)$ type, where $\mathbb{P}_{N}$ is the space of all polynomials of degree less than or equal to $N$ in each space direction. However, as already shown in Sec. 3.3 of [16], these methods are free from any spurious pressure modes, or, equivalently, neither from any algebraic compatibility condition [13], nor from any inf-sup or LBB compatibility condition [1]. Indeed at the boundaries, only Dirichlet conditions are imposed on the velocity, and not the vanishing of its divergence. The pressure is therefore evaluated within a constant.

From now on, $\mathbf{u}$ and $p$ denote the set of the nodal values of the corresponding fields.

\section{STABILITY PROPERTIES OF THE STOKES SOLVERS}

We proceed by an eigenvalue analysis of the fully discrete time evolution operators. For this purpose the source term is discarded $(\mathbf{f}=\mathbf{0})$ and the velocity is constrained to vanish at the boundary.

Let us introduce several discrete operators: $\mathcal{D}$ and $(\mathcal{D}$.) represent, respectively, the gradient and divergence operators; $\mathcal{A}_{D}$ and $\mathcal{A}_{N}$ are defined, respectively, as the discrete Laplacian-Dirichlet and Poisson-Neumann operators; $\mathcal{H}$ is the discrete Helmholtz operator, which includes the homogeneous Dirichlet boundary conditions imposed on the velocity,

$$
\mathcal{H}=\mathcal{A}_{D}-\frac{\gamma_{0}}{\Delta t} \mathcal{I}
$$

and $\mathcal{I}$ is the unit matrix in CCM and a diagonal matrix whose entries are the quadrature weights in SEM.

The time evolution operators for the HOS and HONS methods read:

$$
\mathbf{u}^{n+1}=(\mathcal{H})^{-1}\left[-\frac{\sum_{q=0}^{J_{i}-1} \alpha_{q} \mathbf{u}^{n-q}}{\Delta t}+\mathcal{D} p\right] .
$$

From Eqs. (3.5), (3.6) and (3.14), (3.15), $p$ is solution of the following discrete system:

$$
\begin{aligned}
\mathcal{A}_{N} p & =\mathcal{D} \cdot \mathcal{S}, \\
\frac{\partial p}{\partial n} & =\left(-\sum_{q=0}^{J_{e}-1} \beta_{q} \mathcal{D} \times\left(\mathcal{D} \times \mathbf{u}^{n-q}\right)\right) \cdot \mathbf{n},
\end{aligned}
$$


with $\mathcal{D} \cdot \mathcal{S}$ standing for the discrete right-hand side of the pressure Poisson equations (3.5) and (3.14), respectively, for the HOS and HONS schemes. $\mathcal{S}$ reads then as follows:

$$
\mathcal{S}= \begin{cases}\mathbf{0} & \text { for the HONS scheme, } \\ \frac{\sum_{q=0}^{J_{i}-1} \alpha_{q} \mathbf{u}^{n-q}}{\Delta t} & \text { for the HOS scheme, }\end{cases}
$$

where $\mathcal{S}=\mathbf{0}$ means that the corresponding pressure field comes only from the normal pressure boundary condition.

The resulting evolution matrices are full. Their size is of order $\left[d E J N^{d}\right]^{2}$, where $d$ is the space dimensionality, $J=\max \left(J_{i}, J_{e}\right)$ and $E=1$ for the CCM method. So, their complete spectrum evaluation has been limited to the $2 \mathrm{D}$ case with a reduced number of nodes, namely, $N=24$ (CCM) or $E=2, N=12$ (SEM) for any time order discretisation. For larger nodes numbers either the power method or the ARPACK software [14] is applied to get the leading eigenvalues.

As first results for characterizing the stability properties of the HOS and HONS schemes, the leading eigenvalues of the evolution operators are computed for a large time step, $\Delta t=10^{+7}$, with the CCM $(N=128)$ and SEM $(E=2, N=8)$ discretisations, respectively, for any combination $J_{e}$ and $J_{i}$. For each combination, the leading eigenvalues are found to be identical for the HOS and HONS schemes, in agreement with what can be expected from Eqs. (5.1) and (5.4). Indeed, for very large time step sizes, the normal pressure boundary condition (5.3) fully drives both schemes with $\mathcal{S}=\mathbf{0}$, and the HOS and HONS evolution operators coincide. The real and imaginary parts and the moduli of those eigenvalues are gathered in Tables I and II. The pressure stage time order $J_{e}$ is seen to control the largest eigenvalue magnitude, mainly through the imaginary parts, and therefore the nature of the stability, from the unconditional stability for $J_{e} \leqslant 2$ to a conditional one for $J_{e} \geqslant 3$. In this latter case, a stability criterion on the time step size is expected. It will be commented just below.

The time step size dependence of the leading eigenvalues moduli is depicted in Fig. 1, for the HOS and HONS schemes, with $1 \leqslant J_{e} \leqslant J_{i} \leqslant$ 4 and the $\operatorname{CCM}(N=64)$ discretisation. The schemes with $J_{e}>J_{i}$ are not shown in Fig. 1 for the sake of clarity and because the interesting schemes are when $J_{i} \geqslant J_{e}$ (see Sec. 6). Comparing the $\Delta t=10^{+7}$ data on this figure to those of Tables I and II shows that the leading eigenvalues are almost insensitive to the grid refinement. All the curves of this figure exhibit the same behaviour, namely two plateaus, one at $1-\epsilon, 0<\epsilon \ll 1$, for very small time steps, and another one for large time steps, separated by a transition zone. The former plateau is related to the Euler backward 
Table I. The Leading Eigenvalues $\lambda=\lambda_{r} \pm i \lambda_{i}$ of the HOS- and HONS-CCM $(N=128)$ Evolution Operators for $1 \leqslant\left(J_{e}, J_{i}\right) \leqslant 4$ : The Table Presents $\left|\begin{array}{c}\left(\lambda_{r}, \lambda_{i}\right) \\ |\lambda|\end{array}\right|$

\begin{tabular}{ccccc}
\hline $\begin{array}{c}\text { u-Order } \rightarrow \\
p \text {-order } \downarrow\end{array}$ & $J_{i}=1$ & $J_{i}=2$ & $J_{i}=3$ & $J_{i}=4$ \\
\hline$J_{e}=1$ & $(0.808,0.000)$ & $(0.809,0.000)$ & $(0.809,0.000)$ & $(0.809,0.000)$ \\
& 0.808 & 0.809 & 0.809 & 0.809 \\
$J_{e}=2$ & $(0.809,0.393)$ & $(0.809,0.393)$ & $(0.809,0.393)$ & $(0.809,0.393)$ \\
& 0.899 & 0.899 & 0.899 & 0.899 \\
$J_{e}=3$ & $(0.856,0.783)$ & $(0.856,0.783)$ & $(0.856,0.783)$ & $(0.856,0.783)$ \\
& 1.161 & 1.161 & 1.161 & 1.161 \\
$J_{e}=4$ & $(0.955,1.095)$ & $(0.955,1.095)$ & $(0.955,1.095)$ & $(0.955,1.095)$ \\
& 1.453 & 1.453 & 1.453 & 1.453 \\
\hline
\end{tabular}

Table II. The Leading Eigenvalues $\lambda=\lambda_{r} \pm i \lambda_{i}$ of the HOS- and HONS-SEM ( $E=$ $2, N=12)$ Evolution Operators for $1 \leqslant\left(J_{e}, J_{i}\right) \leqslant 4$ : The Table Presents $\left|\begin{array}{c}\left(\lambda_{r}, \lambda_{i}\right) \\ |\lambda|\end{array}\right|$

\begin{tabular}{ccccc}
\hline $\begin{array}{c}\text { u-Order } \rightarrow \\
p \text {-order } \downarrow\end{array}$ & $J_{i}=1$ & $J_{i}=2$ & $J_{i}=3$ & $J_{i}=4$ \\
\hline$J_{e}=1$ & $(0.860,0.000)$ & $(0.860,0.000)$ & $(0.860,0.000)$ & $(0.860,0.000)$ \\
& 0.860 & 0.860 & 0.860 & 0.860 \\
$J_{e}=2$ & $(0.860,0.347)$ & $(0.860,0.347)$ & $(0.860,0.347)$ & $(0.860,0.347)$ \\
& 0.927 & 0.927 & 0.927 & 0.927 \\
$J_{e}=3$ & $(0.966,0.629)$ & $(0.966,0.629)$ & $(0.966,0.629)$ & $(0.966,0.629)$ \\
& 1.153 & 1.153 & 1.153 & 1.153 \\
$J_{e}=4$ & $(0.995,1.005)$ & $(0.995,1.005)$ & $(0.995,1.005)$ & $(0.995,1.005)$ \\
& 1.414 & 1.414 & 1.414 & 1.414 \\
\hline
\end{tabular}

part of the schemes, the latter one to the explicit extrapolative pressure step, and the transition zone reflects the coupling between these two steps of the schemes. For very small time steps $(\Delta t \rightarrow 0)$, the spectrum of both schemes is clustered nearby the roots of the characteristic polynomial of the backward Euler scheme of order $J_{i}$. These roots are given in Table III, and the leading one is real of magnitude 1 for any $J_{i}$. This explains the plateau at $1-\epsilon$. For both HOS and HONS schemes, the leading eigenvalues moduli are almost insensitive to $J_{i}$, even in the transition zone (see Figs. 2 and 3). For the schemes HOS and HONS, the case $J_{e} \leqslant 2$ (for any $J_{i}$ ) is found to be unconditionally stable for $\Delta t \in\left[10^{-7}, 10^{+7}\right]$, whereas a conditional stability domain appears (at any $J_{i}$ ) with $J_{e} \geqslant 3$. The stability criterion turns out to be of the explicit viscous type due to the pressure 


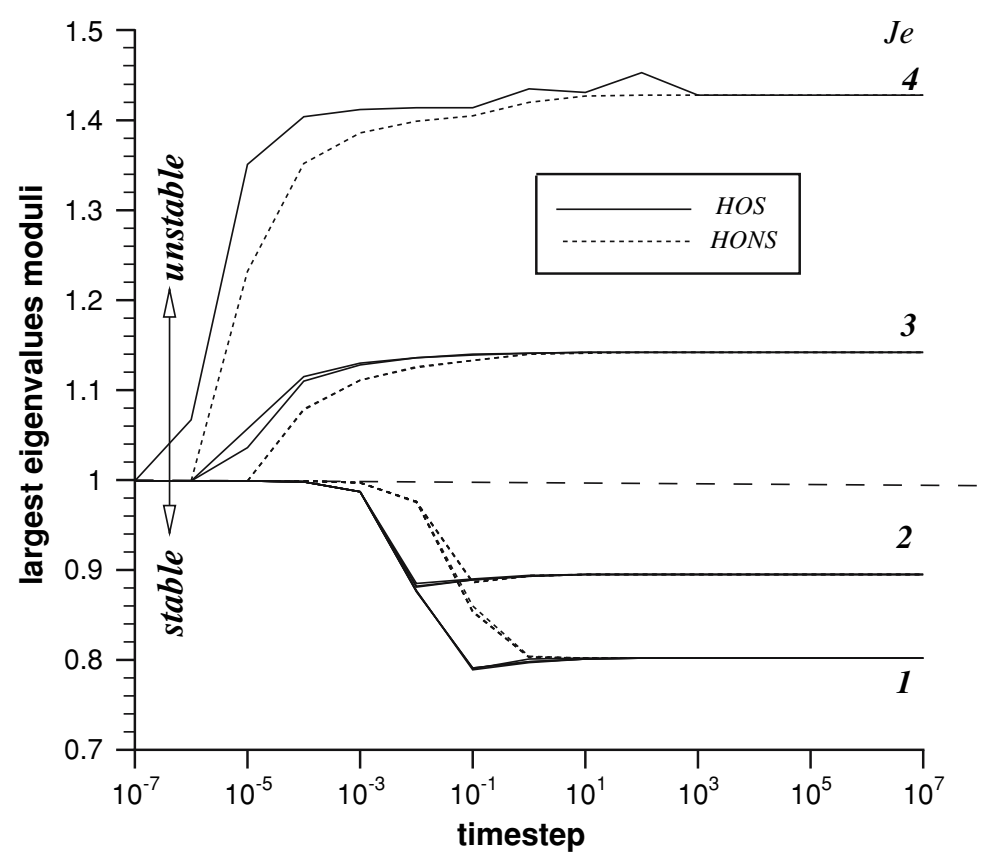

Fig. 1. Time step size dependence of the leading eigenvalues moduli with the CCM $(N=64)$ discretisation and $1 \leqslant J_{e} \leqslant J_{i} \leqslant 4 . J_{i}$ is not indicated for reason appearing in the text.

Table III. The Roots of the Characteristic Polynomial of the Backward Euler Scheme of Order $J_{i}$

\begin{tabular}{lc}
\hline$J_{i}$ & Roots \\
\hline 1 & 1.0 \\
2 & $1.0,1 / 3$ \\
3 & $1.0,(0.3182 \pm 0.2839 \mathrm{i})$ \\
4 & $1.0,(0.2693 \pm 0.492 \mathrm{i}), 0.3815$ \\
\hline
\end{tabular}

Neumann boundary used in the projection stage, $\Delta t<\mathcal{O}\left(N^{-4}\right)$, slightly less stringent for the HONS scheme than for HOS, and also for $J_{e}=3$ compared to $J_{e}=4$. Both these schemes have therefore the nice property of being A-stable up to temporal order 2, in agreement with [5].

It is instructive to have a complete picture of the full spectra of the HOS and HONS evolution operators, for a time step, $\Delta t=10^{-2}$, and any 


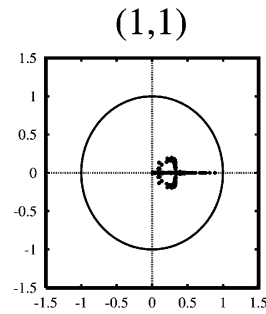

$(2,1)$

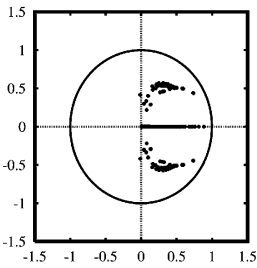

$(3,1)$

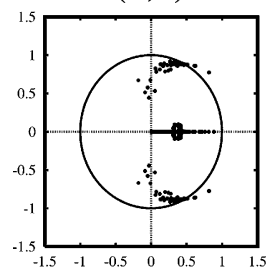

$(4,1)$

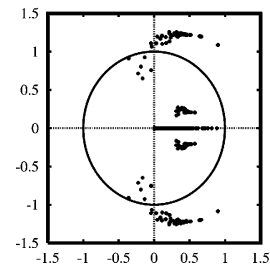

$(1,2)$

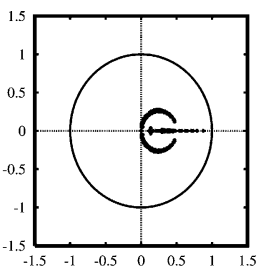

$(2,2)$

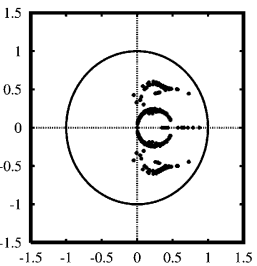

$(3,2)$

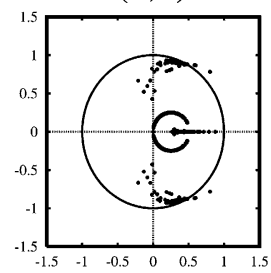

$(4,2)$

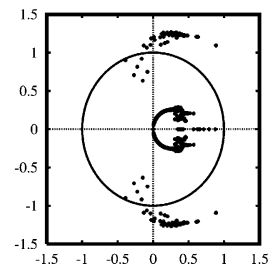

$(1,3)$

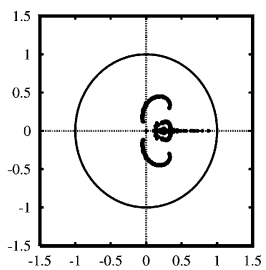

$(2,3)$

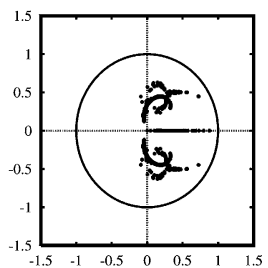

$(3,3)$

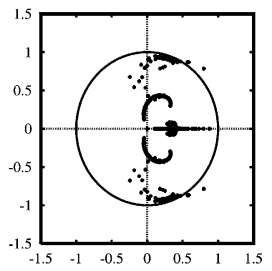

$(4,3)$

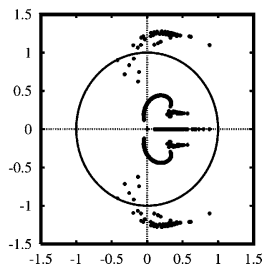

$(1,4)$

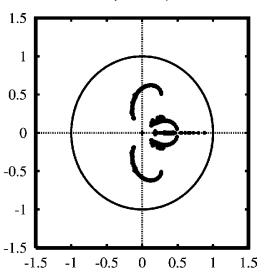

$(2,4)$

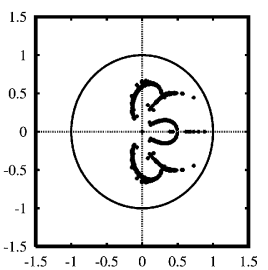

$(3,4)$

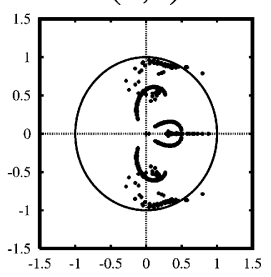

$(4,4)$

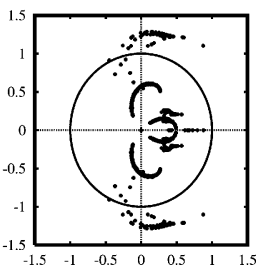

Fig. 2. Full spectra of the HOS-CCM $(N=24)$ evolution operator for a time step $\Delta t=10^{-2}$ and any order combination $1 \leqslant\left(J_{e}, J_{i}\right) \leqslant 4$.

combinations $1 \leqslant\left(J_{e}, J_{i}\right) \leqslant 4$, with the CCM $(N=24)$ and SEM $(E=$ $2, N=12$ ) discretisations. The time step size has been chosen as lying within the corresponding transition zones of Fig. 1. The CCM and SEM applied to the HOS and HONS schemes provide very similar spectra, and for sake of conciseness, only two figures are presented here, Figs. 2 (HOSCCM $(N=24)$ ) and 3 (HONS-SEM $(E=2, N=12)$ ). An increase in the 
$(1,1)$

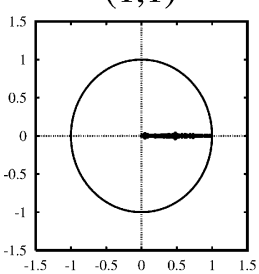

$(2,1)$

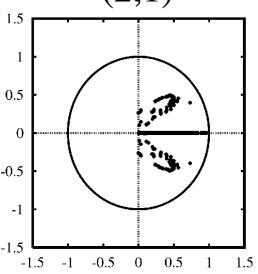

$(3,1)$

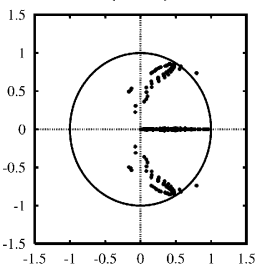

$(4,1)$

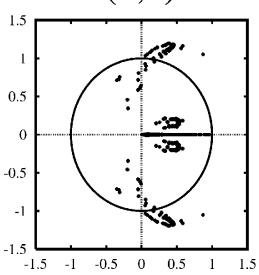

$(1,2)$

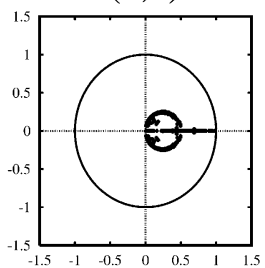

$(2,2)$

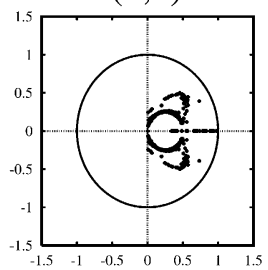

$(3,2)$

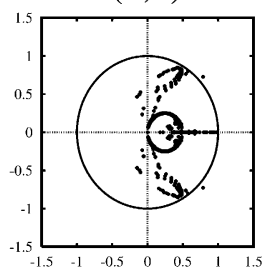

$(4,2)$

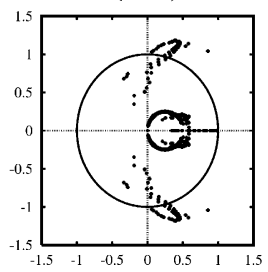

$(1,3)$

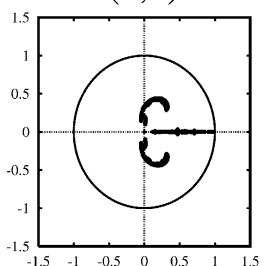

$(2,3)$

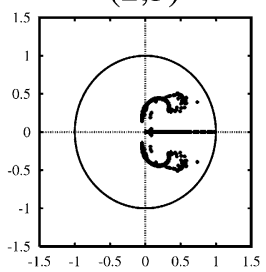

$(3,3)$

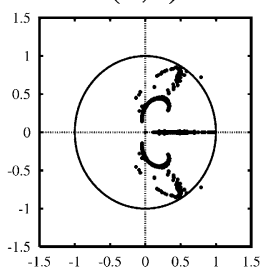

$(4,3)$

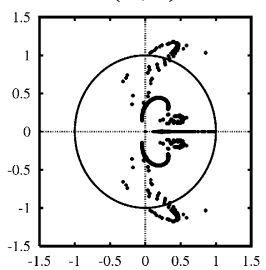

$(1,4)$

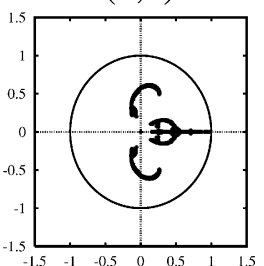

$(2,4)$

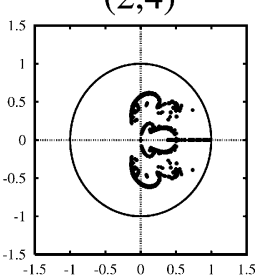

$(3,4)$

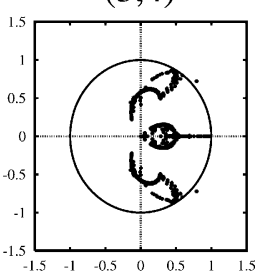

$(4,4)$

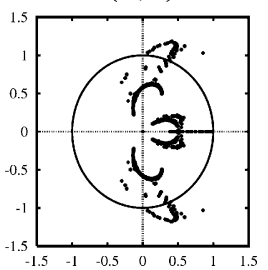

Fig. 3. Full spectra of the HONS-SEM $(E=2, N=12)$ evolution operator for a time step $\Delta t=10^{-2}$ and any order combination $1 \leqslant\left(J_{e}, J_{i}\right) \leqslant 4$.

order $J_{e}$ leads to a spectrum containing larger dominant complex parts, the real parts being almost unaffected, whereas an increase in the order $J_{i}$ makes the spectrum more complex with a more sophisticated structure. For $J_{i} \geqslant 3$, part of this structure is developing in the neighbourhood of the characteristic complex conjugate roots of the BDF polynomial of order $J_{i}$ (see Table III). This part is always included inside the unit circle. 


\section{THE EFFECTIVE TEMPORAL ORDER OF THE STOKES SOLVERS}

A $2 \mathrm{D}$ test problem [2] is considered, in the square $[-1,1]^{2}$, with the source term $\mathbf{f}$ of Eq. (2.1) and the boundary time-dependent velocity $\mathbf{U}$ of Eq. (2.3) analytically chosen so that the following velocity $\mathbf{u}=(u, v)$ and pressure $p$ fields are solutions of the Stokes problem (2.1) and (2.2)

$$
\begin{aligned}
& u(x, y, t)=\sqrt{3} \sin (\sqrt{2} x+t) \cos (\sqrt{3} y+t) \\
& v(x, y, t)=-\sqrt{2} \cos (\sqrt{2} x+t) \sin (\sqrt{3} y+t) \\
& p(x, y, t)=\sqrt{6} \sin (2 x-\sqrt{5} y+0.7 t) \sin (\sqrt{5} y+0.3 t) .
\end{aligned}
$$

The time dependence is periodic, instead of exponentially decreasing, to allow for a non biased experimental confirmation of the stability criteria and identification of the effective temporal orders. The $N=64$ (CCM) and $E=8, N=8$ (SEM) discretisations are taken for evaluating the space derivatives with enough accuracy (almost at the machine limit) to get only temporal errors in the numerical data. The Stokes problem is integrated with the unconditionally stable $\left(J_{e}, J_{i}\right)$ combinations, that is first and second temporal orders in pressure, and first to fourth temporal orders in velocity. The time integration is performed on five units of dimensionless time, with time steps ranging from $10^{-4}$ to $10^{-1}$. The maximum pointwise norm errors are measured for the velocity and pressure with the CCM discretisation, while the Sobolev $H^{1}$ and $L^{2}$ norms are, respectively, considered with the SEM discretisation. Those errors are denoted $\left(E_{\mathbf{u}}(\Delta t), E_{p}(\Delta t)\right)$.

Tables IV-VII give successively the HOS-CCM, HONS-CCM, HOSSEM and HONS-SEM intrinsic accuracies via $\log _{10}\left(\left(E_{\mathbf{u}}(\Delta t), E_{p}(\Delta t)\right)\right)$. This supplies directly the number of correct decimal digits and allows also for a quick evaluation of the effective local orders. Indeed, the errors $\left(E_{\mathbf{u}}(\Delta t), E_{p}(\Delta t)\right)$ are given a phenomenological law proportional to $\Delta t^{\left(\alpha_{\mathbf{u}}, \alpha_{p}\right)}$, with $\left(\alpha_{\mathbf{u}}, \alpha_{p}\right)$ obtained from

$$
\alpha=\frac{\log _{10}\left(E\left(\Delta t_{1}\right) / E\left(\Delta t_{2}\right)\right)}{\log _{10}\left(\Delta t_{1} / \Delta t_{2}\right)}
$$

computed with two successive time steps data. The results are presented in Tables VIII-XI.

Although non-consistent, the HOS scheme appears systematically more accurate, by approximately an order of magnitude on the errors, than the consistent HONS one, for the selected test case. 
Table IV. The Errors $\left(E_{\mathbf{u}}, E_{p}\right)$ on the $(\mathbf{u}, p)$ Fields for the HOS-CCM $(N=64)$ Scheme for any Combination $1 \leqslant J_{i} \leqslant 4(\mathbf{u})$ and $J_{e} \leqslant 2(p)$ : The Table Presents $\left(\log _{10}\left(E_{\mathbf{u}}\right), \log _{10}\left(E_{p}\right)\right)$

\begin{tabular}{cccccccc}
\hline$\Delta t$ & 0.1 & 0.05 & 0.01 & 0.005 & 0.001 & 0.0005 & 0.0001 \\
$\left(J_{i}, J_{e}\right)$ & & & & & & & \\
\hline
\end{tabular}

$\begin{array}{lllllllll}(1,1) & -1.12,0.46 & -1.63,0.27 & -2.78,-0.74 & -3.19,-1.22 & -3.97,-2.38 & -4.26,-2.92 & -4.95,-3.79 \\ (2,1) & -1.30,0.45 & -1.86,0.18 & -3.20,-0.83 & -3.80,-1.30 & -5.17,-2.42 & -5.76,-2.90 & -7.15,-4.03 \\ (3,1) & -1.38,0.41 & -1.94,0.14 & -3.29,-0.88 & -3.88,-1.35 & -5.25,-2.47 & -5.85,-2.95 & -7.23,-4.09 \\ (4,1) & -1.38,0.41 & -1.94,0.14 & -3.29,-0.88 & -3.88,-1.35 & -5.25,-2.47 & -5.85,-2.95 & -7.23,-4.09 \\ (1,2) & -1.96,-0.86 & -2.24,-0.98 & -2.96,-1.89 & -3.26,-2.15 & -3.95,-2.80 & -4.25,-3.09 & -4.95,-3.77 \\ (2,2) & -2.24,-0.19 & -3.05,-0.91 & -4.84,-2.64 & -5.51,-3.38 & -6.98,-5.10 & -7.59,-5.81 & -8.99,-7.45 \\ (3,2) & -2.27,-0.15 & -3.08,-0.91 & -5.08,-2.68 & -5.96,-3.46 & -8.04,-5.26 & -8.93,-6.05 & -11.03,-7.98 \\ (4,2) & -2.31,-0.18 & -3.13,-0.94 & -5.13,-2.71 & -6.01,-3.49 & -8.09,-5.30 & -8.99,-6.08 & -11.08,-8.02\end{array}$

Table V. The Errors $\left(E_{\mathbf{u}}, E_{p}\right)$ on the $(\mathbf{u}, p)$ Fields for the HONS-CCM $(N=64)$ Scheme for any Combination $1 \leqslant J_{i} \leqslant 4(\mathbf{u})$ and $J_{e} \leqslant 2(p)$ : The Table Presents $\left(\log _{10}\left(E_{\mathbf{u}}\right), \log _{10}\left(E_{p}\right)\right)$

\begin{tabular}{cccccccc}
\hline $\begin{array}{c}\Delta t \\
\left(J_{i}, J_{e}\right)\end{array}$ & 0.1 & 0.05 & 0.01 & 0.005 & 0.001 & 0.0005 & 0.0001 \\
\hline$(1,1)$ & $-0.78,0.56$ & $-1.01,0.61$ & $-1.66,0.11$ & $-1.95,-0.17$ & $-2.65,-0.86$ & $-2.95,-1.16$ & $-3.65,-1.86$ \\
$(2,1)$ & $-0.76,0.62$ & $-1.00,0.65$ & $-1.66,0.13$ & $-1.96,-0.16$ & $-2.66,-0.85$ & $-2.96,-1.15$ & $-3.65,-1.85$ \\
$(3,1)$ & $-0.77,0.62$ & $-1.00,0.65$ & $-1.66,0.13$ & $-1.96,-0.16$ & $-2.66,-0.85$ & $-2.96,-1.15$ & $-3.65,-1.85$ \\
$(4,1)$ & $-0.77,0.62$ & $-1.00,0.65$ & $-1.66,0.13$ & $-1.96,-0.16$ & $-2.66,-0.85$ & $-2.96,-1.15$ & $-3.65,-1.85$ \\
$(1,2)$ & $-1.82,-0.38$ & $-2.09,-0.69$ & $-2.64,-1.14$ & $-2.93,-1.41$ & $-3.61,-2.09$ & $-3.91,-2.38$ & $-4.61,-3.08$ \\
$(2,2)$ & $-1.53,0.25$ & $-2.21,-0.69$ & $-3.66,-2.18$ & $-4.26,-2.77$ & $-5.67,-4.16$ & $-6.27,-4.76$ & $-7.71,-6.38$ \\
$(3,2)$ & $-1.60,0.13$ & $-2.29,-0.71$ & $-3.74,-2.02$ & $-4.35,-2.61$ & $-5.75,-4.00$ & $-6.36,-4.61$ & $-7.81,-6.14$ \\
$(4,2)$ & $-1.61,0.12$ & $-2.29,-0.72$ & $-3.74,-2.02$ & $-4.35,-2.61$ & $-5.75,-4.00$ & $-6.36,-4.61$ & $-7.81,-6.14$ \\
\hline
\end{tabular}

\section{Moreover,}

(a) from the inspection of the columns corresponding to $\Delta t \leqslant 0.01$, the last four columns in Tables VIII-XI, a fairly good uniformity of the local exponents $\left(\alpha_{\mathbf{u}}, \alpha_{p}\right)$ allows us to define the effective time orders of both schemes. They are summarized, rounded in $1 / 2$ multiples, in Table XII. The HONS scheme is fully controlled, as expected, by the lowest order among $J_{i}$ and $J_{e}$, leading to $\alpha_{\mathbf{u}}=\alpha_{p}=\min \left(J_{i}, J_{e}\right)$. For the HOS scheme, no such simple law appears, except in the interesting cases where $J_{i} \geqslant J_{e}$ for which the pressure order is $\alpha_{p}=J_{e}+1 / 2$. As regards the 
Table VI. The Errors $\left(E_{\mathbf{u}}, E_{p}\right)$ on the $(\mathbf{u}, p)$ Fields for the HOS-SEM $(E=8, N=8)$ Scheme for any Combination $1 \leqslant J_{i} \leqslant 4(\mathbf{u})$ and $J_{e} \leqslant 2(p)$ : The Table Presents $\left(\log _{10}\left(E_{\mathbf{u}}\right), \log _{10}\left(E_{p}\right)\right)$

\begin{tabular}{cccccccc}
\hline$\Delta t$ & 0.1 & 0.05 & 0.01 & 0.005 & 0.001 & 0.0005 & 0.0001 \\
$\left(J_{i}, J_{e}\right)$ & & & & & & & \\
\hline
\end{tabular}

$\begin{array}{llllllll}(1,1) & -1.36,0.06 & -1.87,-0.26 & -3.00,-1.31 & -3.41,-1.81 & -4.21,-3.06 & -4.50,-3.55 & -5.19,-4.20 \\ (2,1) & -1.54,0.02 & -2.09,-0.33 & -3.42,-1.40 & -4.01,-1.89 & -5.40,-3.04 & -6.00,-3.54 & -7.38,-4.72 \\ (3,1) & -1.62,-0.03 & -2.16,-0.38 & -3.50,-1.45 & -4.10,-1.94 & -5.49,-3.09 & -6.08,-3.60 & -7.47,-4.77 \\ (4,1) & -1.66,-0.05 & -2.21,-0.41 & -3.56,-1.49 & -4.15,-1.98 & -5.55,-3.13 & -6.14,-3.63 & -7.52,-4.81 \\ & & & & & & & \\ (1,2) & -2.19,-1.30 & -2.48,-1.69 & -3.20,-2.30 & -3.49,-2.56 & -4.19,-3.21 & -4.49,-3.50 & -5.19,-4.18 \\ (2,2) & -2.42,-0.74 & -3.23,-1.45 & -5.08,-3.18 & -5.75,-3.93 & -7.22,-5.63 & -7.83,-6.34 & -9.23,-7.89 \\ (3,2) & -2.41,-0.71 & -3.23,-1.47 & -5.25,-3.26 & -6.15,-4.04 & -8.24,-5.89 & -9.14,-6.69 & -11.24,-8.57 \\ (4,2) & -2.45,-0.75 & -3.28,-1.50 & -5.30,-3.29 & -6.20,-4.07 & -8.29,-5.92 & -9.20,-6.73 & -11.27,-8.60\end{array}$

Table VII. The Errors $\left(E_{\mathbf{u}}, E_{p}\right)$ on the $(\mathbf{u}, p)$ Fields for the HONS-SEM $(E=8, N=8)$ Scheme for any Combination $1 \leqslant J_{i} \leqslant 4(\mathbf{u})$ and $J_{e} \leqslant 2(p)$ : The Table Presents $\left(\log _{10}\left(E_{\mathbf{u}}\right), \log _{10}\left(E_{p}\right)\right)$

\begin{tabular}{cccccccc}
\hline $\begin{array}{c}\Delta t \\
\left(J_{i}, J_{e}\right)\end{array}$ & 0.1 & 0.05 & 0.01 & 0.005 & 0.001 & 0.0005 & 0.0001 \\
\hline
\end{tabular}

$\begin{array}{llllllllll}(1,1) & -1.00,0.12 & -1.25,0.15 & -1.90,-0.42 & -2.19,-0.70 & -2.89,-1.39 & -3.19,-1.69 & -3.89,-2.39 \\ (2,1) & -1.00,0.23 & -1.25,0.17 & -1.90,-0.40 & -2.20,-0.69 & -2.89,-1.38 & -3.19,-1.68 & -3.89,-2.38 \\ (3,1) & -1.02,0.29 & -1.24,0.17 & -1.90,-0.40 & -2.20,-0.69 & -2.89,-1.38 & -3.19,-1.68 & -3.89,-2.38 \\ (4,1) & -1.00,0.22 & -1.24,0.17 & -1.90,-0.40 & -2.20,-0.69 & -2.89,-1.38 & -3.19,-1.68 & -3.89,-2.38 \\ & & & & & & & & & \\ (1,2) & -2.05,-0.94 & -2.33,-1.29 & -2.88,-1.70 & -3.16,-1.97 & -3.85,-2.65 & -4.15,-2.94 & -4.85,-3.64 \\ (2,2) & -1.73,-0.33 & -2.42,-1.25 & -3.88,-2.71 & -4.49,-3.29 & -5.90,-4.68 & -6.50,-5.28 & -7.90,-6.68 \\ (3,2) & -1.80,-0.46 & -2.51,-1.25 & -4.59,-3.14 & -3.98,-2.55 & -5.99,-4.53 & -6.59,-5.13 & -7.99,-6.53 \\ (4,2) & -1.81,-0.47 & -2.52,-1.25 & -3.98,-2.55 & -4.59,-3.14 & -5.99,-4.53 & -6.59,-5.13 & -7.99,-6.53\end{array}$

velocity time order, it can be noticed that the highest unconditionally stable order is 3 , provided that $J_{e}=2$;

(b) for larger $\Delta t$ 's, the local time orders depart significantly from the previous ones and do not lend themselves to an easy interpretation.

In [11], Guermond and Shen proved the stability and convergence of the HOS $J_{i}=1,2$ and $J_{e}=1$ scheme. The velocity and pressure error estimates are in full agreement with the present study (see Table XII). 
Table VIII. Local $(\mathbf{u}, p)$ Time Accuracy Orders $\left(\alpha_{\mathbf{u}}, \alpha_{p}\right)$ for the HOS-CCM $(N=64)$ Scheme for any Combination $1 \leqslant J_{i} \leqslant 4(\mathbf{u})$ and $J_{e} \leqslant 2(p)$

\begin{tabular}{ccccccc}
\hline $\begin{array}{c}\Delta t \in \\
\left(J_{i}, J_{e}\right)\end{array}$ & {$[0.1,0.05]$} & {$[0.05,0.01]$} & {$[0.01,0.005]$} & {$[0.005,0.001]$} & {$[0.001$,} & {$[0.0005$,} \\
$0.0005]$ & $0.00001]$ \\
\hline$(1,1)$ & $1.67,0.64$ & $1.64,1.44$ & $1.38,1.60$ & $1.11,1.67$ & $0.97,1.79$ & $0.99,1.25$ \\
$(2,1)$ & $1.84,0.88$ & $1.92,1.45$ & $1.97,1.58$ & $1.96,1.59$ & $1.97,1.60$ & $1.98,1.62$ \\
$(3,1)$ & $1.85,0.91$ & $1.93,1.45$ & $1.98,1.59$ & $1.96,1.60$ & $1.97,1.61$ & $1.98,1.62$ \\
$(4,1)$ & $1.85,0.91$ & $1.93,1.45$ & $1.98,1.59$ & $1.96,1.60$ & $1.97,1.61$ & $1.98,1.62$ \\
$(1,2)$ & $0.92,0.40$ & $1.03,1.31$ & $0.98,0.86$ & $0.99,0.93$ & $1.00,0.96$ & $1.00,0.98$ \\
$(2,2)$ & $2.70,2.38$ & $2.56,2.47$ & $2.24,2.48$ & $2.09,2.45$ & $2.03,2.39$ & $2.01,2.34$ \\
$(3,2)$ & $2.71,2.52$ & $2.85,2.54$ & $2.94,2.56$ & $2.97,2.59$ & $2.99,2.61$ & $3.00,2.76$ \\
$(4,2)$ & $2.71,2.52$ & $2.86,2.54$ & $2.94,2.57$ & $2.97,2.59$ & $2.99,2.62$ & $3.00,2.77$ \\
\hline
\end{tabular}

Table IX. Local $(\mathbf{u}, p)$ Time Accuracy Orders $\left(\alpha_{\mathbf{u}}, \alpha_{p}\right)$ for the HONS-CCM $(N=64)$ Scheme for any Combination $1 \leqslant J_{i} \leqslant 4(\mathbf{u})$ and $J_{e} \leqslant 2(p)$

\begin{tabular}{ccccccc}
\hline $\begin{array}{c}\Delta t \in \\
\left(J_{i}, J_{e}\right)\end{array}$ & {$[0.1,0.05]$} & {$[0.05,0.01]$} & {$[0.01,0.005]$} & {$[0.005,0.001]$} & {$[0.001$,} & {$[0.0005$,} \\
$0.0005]$ & $0.00001]$ \\
\hline$(1,1)$ & $0.76,-0.16$ & $0.93,0.72$ & $0.98,0.93$ & $0.99,0.98$ & $1.00,1.00$ & $1.00,1.00$ \\
$(2,1)$ & $0.80,-0.10$ & $0.95,0.75$ & $0.99,0.96$ & $1.00,0.99$ & $1.00,1.00$ & $1.00,1.00$ \\
$(3,1)$ & $0.79,-0.11$ & $0.94,0.75$ & $0.99,0.96$ & $1.00,0.99$ & $1.00,1.00$ & $1.00,1.00$ \\
$(4,1)$ & $0.79,-0.11$ & $0.94,0.74$ & $0.99,0.96$ & $1.00,0.99$ & $1.00,1.00$ & $1.00,1.00$ \\
$(1,2)$ & $0.92,1.03$ & $0.78,0.65$ & $0.95,0.90$ & $0.98,0.97$ & $1.00,0.99$ & $1.00,1.00$ \\
$(2,2)$ & $2.24,3.14$ & $2.07,2.13$ & $2.02,1.95$ & $2.01,1.98$ & $2.01,2.01$ & $2.06,2.31$ \\
$(3,2)$ & $2.29,2.81$ & $2.08,1.87$ & $2.02,1.97$ & $2.01,1.99$ & $2.01,2.01$ & $2.08,2.20$ \\
$(4,2)$ & $2.26,2.77$ & $2.08,1.86$ & $2.02,1.97$ & $2.01,1.99$ & $2.01,2.01$ & $2.08,2.20$ \\
\hline
\end{tabular}

\section{CONCLUSIONS}

We investigated the spectral discretization of the unsteady Stokes problem through a CCM and a Legendre SEM. Velocity and pressure computations are decoupled by two splitting schemes. The pressure is evaluated through an extrapolated in time (of theoretical order $J_{e}$ ) Neumann boundary condition and the velocity is obtained by a time implicit (backward Euler scheme of theoretical order $J_{i}$ ) diffusion step. The first scheme is HOS scheme while the second one is a HONS scheme known to be a consistent split scheme. The common result is that the schemes are 
Table X. Local $(\mathbf{u}, p)$ Time Accuracy Orders $\left(\alpha_{\mathbf{u}}, \alpha_{p}\right)$ for the HOS-SEM $(E=8, N=8)$ Scheme for any Combination $1 \leqslant J_{i} \leqslant 4(\mathbf{u})$ and $J_{e} \leqslant 2(p)$

\begin{tabular}{|c|c|c|c|c|c|c|}
\hline $\begin{array}{c}\Delta t \in \\
\left(J_{i}, J_{e}\right)\end{array}$ & {$[0.1,0.05]$} & {$[0.05,0.01]$} & {$[0.01,0.005]$} & {$[0.005,0.001]$} & {$[0.001,0.0005]$} & $\begin{array}{l}{[0.0005,} \\
0.00001]\end{array}$ \\
\hline$(1,1)$ & $1.67,1.07$ & $1.63,1.50$ & $1.37,1.66$ & $1.13,1.79$ & $0.97,1.63$ & $0.99,0.93$ \\
\hline$(2,1)$ & $1.81,1.16$ & $1.91,1.53$ & $1.97,1.62$ & $1.99,1.64$ & $1.98,1.67$ & $1.98,1.69$ \\
\hline$(3,1)$ & $1.81,1.17$ & $1.92,1.53$ & $1.97,1.62$ & $1.99,1.65$ & $1.97,1.67$ & $1.98,1.69$ \\
\hline$(4,1)$ & $1.83,1.19$ & $1.92,1.54$ & $1.98,1.63$ & $1.99,1.65$ & $1.97,1.67$ & $1.98,1.69$ \\
\hline$(1,2)$ & $0.96,1.32$ & $1.03,0.87$ & $0.98,0.86$ & $0.99,0.93$ & $1.00,0.96$ & $1.00,0.98$ \\
\hline$(2,2)$ & $2.69,2.38$ & $2.65,2.48$ & $2.23,2.49$ & $2.09,2.44$ & $2.03,2.34$ & $2.01,2.21$ \\
\hline$(3,2)$ & $2.72,2.50$ & $2.89,2.56$ & $2.97,2.61$ & $2.99,2.64$ & $3.00,2.67$ & $3.00,2.69$ \\
\hline$(4,2)$ & $2.73,2.50$ & $2.90,2.56$ & $2.98,2.61$ & $2.99,2.64$ & $3.00,2.67$ & $2.97,2.68$ \\
\hline
\end{tabular}

Table XI. Local $(\mathbf{u}, p)$ Time Accuracy Orders $\left(\alpha_{\mathbf{u}}, \alpha_{p}\right)$ for the HONS-SEM $(E=8, N=8)$ Scheme for any Combination $1 \leqslant J_{i} \leqslant 4(\mathbf{u})$ and $J_{e} \leqslant 2(p)$

\begin{tabular}{ccccccc}
\hline $\begin{array}{c}\Delta t \in) \\
\left(J_{i}, J_{e}\right)\end{array}$ & {$[0.1,0.05]$} & {$[0.05,0.01]$} & {$[0.01,0.005]$} & {$[0.005,0.001]$} & {$[0.001,0.0005]$} & {$[0.0005$,} \\
& & & & & & $0.00001]$ \\
\hline$(1,1)$ & $0.84,-0.08$ & $0.93,0.80$ & $0.98,0.95$ & $0.99,0.99$ & $1.00,1.00$ & $1.00,1.00$ \\
$(2,1)$ & $0.85,0.20$ & $0.92,0.81$ & $0.99,0.97$ & $1.00,0.99$ & $1.00,1.00$ & $1.00,1.00$ \\
$(3,1)$ & $0.74,0.38$ & $0.94,0.81$ & $0.99,0.97$ & $1.00,0.99$ & $1.00,1.00$ & $1.00,1.00$ \\
$(4,1)$ & $0.78,0.16$ & $0.94,0.81$ & $0.99,0.97$ & $1.00,0.99$ & $1.00,1.00$ & $1.00,1.00$ \\
$(1,2)$ & $0.92,1.16$ & $0.79,0.60$ & $0.95,0.89$ & $0.98,0.96$ & $1.00,0.99$ & $1.00,1.00$ \\
$(2,2)$ & $2.31,3.05$ & $2.09,2.08$ & $2.03,1.95$ & $2.01,1.98$ & $2.00,1.99$ & $2.00,2.00$ \\
$(3,2)$ & $2.38,2.63$ & $2.08,1.89$ & $2.02,1.97$ & $2.01,1.98$ & $2.00,2.00$ & $2.00,2.00$ \\
$(4,2)$ & $2.37,2.59$ & $2.09,1.86$ & $2.02,1.97$ & $2.01,1.99$ & $2.00,2.00$ & $2.00,2.00$ \\
\hline
\end{tabular}

unconditionally stable for $J_{e} \leqslant 2$, whereas a time step criterion of explicit type occurs $-\Delta t<\mathcal{O}\left(N^{-4}\right)$-, when $J_{e}>2$, slightly less restrictive for the consistent scheme than for the other. The effective time orders are then measured with $1 \leqslant J_{e} \leqslant 2$ and $1 \leqslant J_{i} \leqslant 4$. For the consistent solver the effective and expected time orders are in excellent agreement, and some discrepancies occur for the other scheme which turns out to provide slightly more accurate orders. Finally, the $\operatorname{HOS}\left(J_{i}=3, J_{e}=2\right)$ unconditionally stable scheme provides the best measured time orders, namely, 3rd order for the velocity and 2.5 th order for the pressure. 
Table XII. The Effective Time Orders of the HOS and HONS Schemes with the CCM $(N=64)$ or SEM $(E=8, N=8)$ Discretisations for $1 \leqslant J_{i} \leqslant 4$ and $J_{e} \leqslant 2$ : The Table Presents

$$
\left|\begin{array}{c}
\left(\alpha_{\mathbf{u}}, \alpha_{p}\right)-\text { HOS } \\
\left(\alpha_{\mathbf{u}}, \alpha_{p}\right)-\text { HONS }
\end{array}\right|
$$

\begin{tabular}{ccccc}
\hline $\begin{array}{c}\mathbf{u} \text {-order } \rightarrow \\
p \text {-order } \downarrow\end{array}$ & $J_{i}=1$ & $J_{i}=2$ & $J_{i}=3$ & $J_{i}=4$ \\
\hline$J_{e}=1$ & $(1,3 / 2)$ & $(2,3 / 2)$ & $(2,3 / 2)$ & $(2,3 / 2)$ \\
& $(1,1)$ & $(1,1)$ & $(1,1)$ & $(1,1)$ \\
$J_{e}=2$ & $(1,1)$ & $(2,5 / 2)$ & $(3,5 / 2)$ & $(3,5 / 2)$ \\
& $(1,1)$ & $(2,2)$ & $(2,2)$ & $(2,2)$ \\
\hline
\end{tabular}

\section{ACKNOWLEDGMENTS}

The authors would like to thank Prof. J. Shen and Dr. J.L. Guermond for helpful discussions. The second author gratefully acknowledges the financial support of the Fonds National Suisse de la Recherche Scientifique under Grant FN 21-61792.00. The third author received financial support from the ERCOFTAC visitor programme sponsored by the L. Euler Pilot Center (Switzerland) at EPFL. The computing resources were made available by CSCS, Manno, Switzerland.

\section{REFERENCES}

1. Auteri, F., Guermond, J. L., and Parolini, N. (2001). Role of the LBB condition in weak spectral projection methods. J. Comput. Phys. 174, 405-420.

2. Batoul, A., Khallouf, H., and Labrosse, G. (1994). Une méthode de résolution directe (Pseudo-Spectrale) du problème de Stokes 2D/3D instationnaire. Application à la cavité entrainée carrée. C.R. Acad. Sci. Paris 319 Série I, 1455-1461.

3. Canuto, C., Hussaini, M. Y., Quarteroni, A., and Zang, T. A. (1988). Spectral Methods in Fluid Dynamics, Springer Series in Computational Physics, Springer-Verlag, New-York.

4. Chorin, A. J., (1968). Numerical solution of the Navier-Stokes equations. Math. Comp. 22, 745-761.

5. Dahlquist, G. (1963). A special stability problem for linear multistep methods. BIT 3, 2743.

6. Deville, M. O., Fischer, P. F., and Mund, E. H., (2002). High-Order Methods for Incompressible Fluid Flow, Cambridge University Press, New-York.

7. Dubois-Pélerin, Y., van Kemenade, V., and Deville, M. O. (1999). An object oriented toolbox for spectral element analysis. J. Sci. Comput. 14, 1-29.

8. Gottlieb, D. and Orszag, S. A. (1977). Numerical Analysis of Spectral Methods: Theory and Applications. SIAM-CBMS, Philadelphia.

9. Guermond, J. L. and Shen, J. Quelques résultats nouveaux sur les méthodes de projection (2001). C.R. Acad. Sci. Paris 333 Série I, 1111-1116. 
10. Guermond, J. L. and Shen, J. (2003). A new class of truly consistent splitting schemes for incompressible flows. J. Comput. Phys. 192, 262-276.

11. Guermond, J. L. and Shen, J. (2003). Velocity-correction projection methods for incompressible flows. Siam J. Numer. Anal. 41(1), 112-134.

12. Karniadakis, G. E. M., Israeli, M., and Orszag, S. A. (1991). High-order splitting methods for the incompressible Navier-Stokes equations. J. Comput. Phys. 97, 414-443.

13. Labrosse, G. (1993). Compatibility conditions for the Stokes system discretized in 2D Cartesian domains. Comput. Meth. Appl. Mech. Eng. 106, 353-365.

14. Lehoucq, R. B., Sorensen, D. C., and Yang, C. (1998). ARPACK Users' Guide. SIAM, Philadelphia.

15. Leriche, E. and Gavrilakis, S. (2000). Direct numerical simulation of the flow in a liddriven cubical cavity. Phys. Fluids 12(6), 1363-1376.

16. Leriche, E. and Labrosse, G. (2000). High-order direct Stokes solvers with or without temporal splitting: Numerical investigations of their comparative properties. SIAM J. Scient. Comput. 22(4), 1386-1410.

17. Orszag, S. A., Israeli, M., and Deville, M. O. (1986). Boundary conditions for incompressible flows. J. Sci. Comput. 1(1), 75-111.

18. Témam, R. (1969). Sur l'approximation de la solution de Navier-Stokes par la méthode des pas fractionnaires. I, II. Arch. Rat. Mech. Anal. 32, 135-153, 377-385. 